\title{
Using Dependency and Structure Modeling for temporal decision making in Set-Based Design
}

\author{
Stephen Rapp ${ }^{1}$, Gary Witus ${ }^{2}$, Shreya Kalgave ${ }^{2}$ \\ ${ }^{1}$ U.S. Army AFC, CCDC - Ground Vehicle Systems Center, Warren, Michigan \\ ${ }^{2}$ Wayne State University, Detroit, Michigan
}

\begin{abstract}
Set-Based Design (SBD) is a Product Development (PD) methodology that defines a set of system designs to reduce the uncertainty associated with point designs. SBD explores trade-offs and searches for set solutions that balance risk and performance. SBD temporally down selects the design as uncertainty clears at epochs. Dependency and Structure Modeling is a process allowing structured model representation of a system and dependencies. PD is epoch based. Defense PD is particularly vulnerable to requirements creep and budget uncertainty. We propose integrating Dependency and Structure Modeling to support SBD PD by highlighting system dependencies to improve SBD design down selection. We provide a literature review of Dependency and Structure Modeling that potentially supports SBD in PD. We present a police/military PD example to showcase a potential framework temporally integrating Dependency and Structure Modeling into SBD PD.
\end{abstract}

Dependency and Structure Modeling, Design Structure Matrix (DSM), Set-Based Design (SBD), Product Development (PD), Defense, stick camera

\section{Introduction:}

\section{What is SBD?}

(Singer, et.al. 2009) SBD is a PD design selection process that considers a set of design alternatives to maintain through PD while risk and uncertainty are reduced. The set of design alternatives is temporally reduced to an eventual point or small subset of point design(s) required for manufacturing a system or family of systems. (Sobek, et.al. 1999) Toyota developed SBD as a Concurrent Engineering sub-process in its Production System. SBD also reduces design cycling with evolving requirements. The underlying principle is delayed differentiation: making decisions that enable future decision options.

\section{What is Dependency and Structured Modeling and how it is used?}

Dependency and Structure Modeling is a process that allows to look at a system's product or process structures or elements and their dependencies. The DSM tool encapsulates those relationships and dependencies in its matrix. The tool represents systems and allows matrix analyses, such as clustering and sequencing. While there are many DSM types, we will use the Product DSM to represent technology options in our example. The DSM represents the information flow and the clustering informs PD engineers and managers where system integration complexity and risk reside. In turn, this informs us what the best set solution is forward from design to production. We will use DSM throughout PD, but focus it to support, review and understand element selections at PD epochs! This framework process is below in Figure 1. 


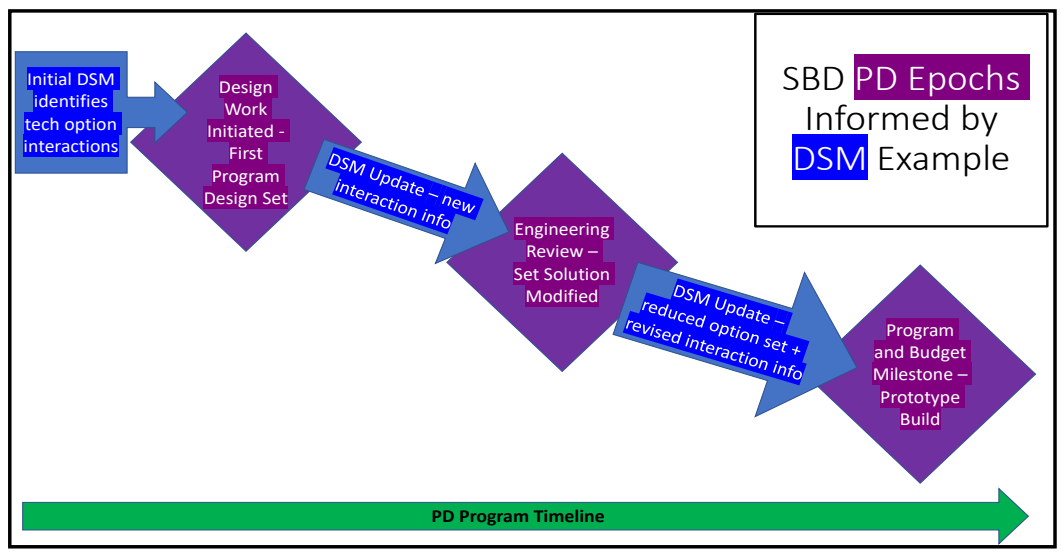

Figure 1: SBD Epochal Framework Informed by DSM

\section{Literature Review Insights:}

(McKenny, et.al. 2011) presents an application of SBD for Navy ship design focusing on adapting to requirement changes. They developed and simulated cycles of SBD designs. They provided a structured approach to evaluate design space and make informed resilient decisions. Their results showed that requirement changes can be handled by SBD. The results of their experiment were in the form of reduced sets solutions over time. Although the experiment provided good insights on how to use SBD, it did not include any implementation of DSM or any usage of the tool.

(Browning 2015) and (Doerry 2009) discuss DSM usage. Browning's article provides insights into DSM, addressing static and temporal DSM models that offer possibilities for visualizing, analyzing, and improving PD tools. Doerry's discussion suggested different design approaches and design activities to leverage DSM. Conceptually, he extended exploration of alternate designs to decouple design activities for optimized complex design projects. DSM's use to decompose system integration complexity while tracking and updating dependencies is insightful. Furthermore, these ideas contribute to identifying complexities earlier.

(Rapp, et.al. 2018) The PD process is complex due to design decisions being nearly impossible to optimize while technology and integration are unknown early in the process. He presents a quantitative framework that values design trajectory actions resiliently through time in PD. Subsystem tech options are considered to frame initial system sets that taper over time to a final smaller set. A quantitative SBD framework was proposed where a Markov Decision Process defines design value epoch to epoch. The framework optimizes the design(s) subject to budget, recursively solving the problem with dynamic programming at each epoch. (Rapp, 2017) proposed the use of DSM with SBD to review PD decisions with updated DSM information at the epochs.

The literature review provided us a core foundation to explore using the DSM tool to better execute SBD temporally in PD, but the body of knowledge is sparse. 


\section{Example Problem: Police Endoscope Camera (PEC) "Camera on a stick":}

Police/Special Response Teams deal with situations where they need to use a camera device system to monitor the situation without any disturbance. This device should allow the user to be unobtrusive, to insert from the adjacent room, to collapse on a belt holster, to assemble quickly and reliably, be able to be inserted through narrow gaps, be effective in unlit low-light conditions, and have a wide field of view. Special Response Teams had tried using commercial endoscopes designed for plumbing and construction, but they were unsuitable as they were designed for very short-range inspection.

Traditional point-based PD for such a system will not typically produce a globally optimal design with richness in trade space. Design methods such as Set-Based Design (SBD) provide a structural approach to evaluate design system trade space and uncertainty to make informed decisions through the PD. DSM supports a reduction in uncertainty by considering the product dependencies. System integration, especially with newer technology options, always carries great uncertainty. The example problem has a smaller number of subsystems and technology options whereby we can consider these points.

PD involves a huge commitment of time and resources. In this study, we propose injecting the usage of DSM in the SBD process temporally to support the epochal decisions. By using DSM throughout PD, the design optimization and PD itself can benefit by increasing a sharper view and context into the system integration which is directly tied to the product technology dependencies. This logic will be explored in the PEC example.

System alternatives are created and then considered for development based on the possible combinations of the subsystem technology options available. Each epoch requires a decision to reduce or maintain, or possibly even expand the set of technology options for development based. We propose that maintaining a DSM matrix will help with the epochal design decisions. Designing any product takes time and cost, and it depends upon the technology and design which are chosen based on the availability and viability of the technology options in the trade space. The understanding of the dependencies between those options, from DSM, can focus the epochal decisions themselves.

During development, identifying a manageable set of design parameters that have been determined to be principal factors in achieving maximum design value plays a key role. DSM tool helps with determining which of the set is more important than the others by identifying which design attributes and measures are most important in differentiating among the most promising design combinations. This approach can be tied to Acquisition Epochs/Milestones, as shown in Figure 2. 


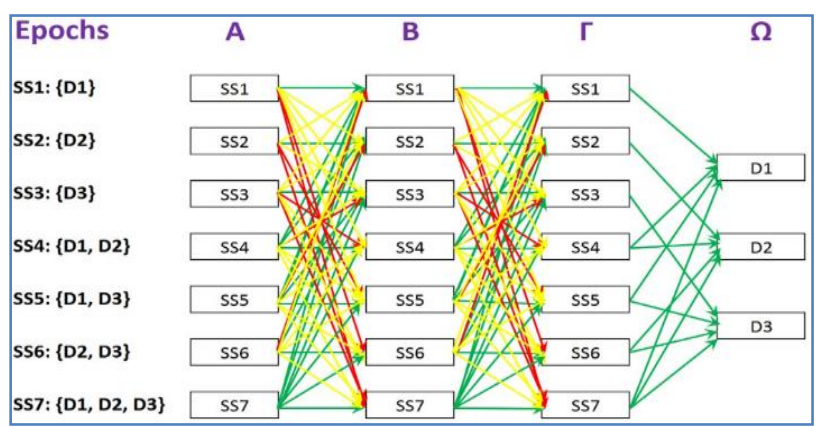

SS: Set Solution

D: A Single, Point Design

The colored arcs

represent one (yellow) or two (red) design catchups not developed in the previous epoch. Green represents no catchup required.

Figure 2: Analytical Set-Based Design Process tied to Acquisition Epochs/Milestones

These decisions can be based on qualitative data, compatibility, trade-space, and future changes in operational requirements, technology projections, program budgets, and other changes in the design environment. Our target is to choose a better system option or to reject available options to achieve a set of designs with a variety of combinations.

\section{Key Performance Parameter (KPP):}

KPPs represent capabilities or characteristics so that failure to meet the threshold of performance can cause system failure. KPPs are quantifiable outcomes that must be met for a system to meet its operational goals. The anatomy of structured KPPs includes:

A Measure - ALL KPPs must be measurable.

A Target - Every KPP must have a target value. KPPs have threshold and objective values. The difference between objective and threshold values set the trade space.

The following are the various situational needs of a camera system that might arise in various police/military operations:

1. Seeing underneath any object (vehicles, rooms, doors, etc.)

2. Inspecting hazardous areas from a specific distance:

3. Observing and investigating Dark and/or Hidden Areas:

4. The need for reliable extension pole with multiple segments:

The KPPs were decided based on situations that can be faced by the police or respective department using these cameras.

\section{List of police \& military situations and camera-pole system applications:}

1. Hostage situations (Hostile numbers, run, hide, rapidly respond to a specific location)

2. Hard to reach areas where human reach is nearly impossible (Hidden dark areas, underneath of vehicles, complex narrow structures, etc.)

3. To prevent suspected explosions, bombing at a specific location and to investigate other suspecting items or vehicles for civilian's safety

4. To conduct rescue operation (Active shooters, snipers, access the victim, civilian's surveillance, etc.) 
5. Based on the situations that often arise in police and military, we listed out the most critical KPPs with their essential features.

In our problem, we have considered three subsystems to develop the camera on a stick system: extension rod, camera system, electronic interface of the camera.

\section{CAMERA:}

Low light sensitivity: Low light sensitivity depends on factors ranging from the size and light transmission capability of the sensor element, signal to the noise of camera amplifier, and shutter speed. Night vision enables us to see up to 30 feet in absolute darkness.

Video Recording Quality: 720*576 pixels which is also known as D1 quality recording for better visualization.

Camera working Time: This is an essential part of our complete system as we might need a moderate to a good amount of battery power for both standby and working time.

Size: The size of the camera is an important factor as the smaller and compacter the size, less the time of handling and other factors which are carrying and opening time.

Charging time: The compact battery needs frequent charging for high-resolution audio/video recording in daylight and night hours. Large batteries up to 3600mAH (12 hours audio/video) need more space. Compactness and charge are tradeable.

Field of View (FOV): The requirement for FOV (field of view) as the visible area or length is useful to define in a design of PEC system based on the requirement whether it is intended to use at a single specified working distance or different working distances (requires a large field of view and a relatively long depth of field).

Motion detection: It is a critical factor that fulfills the core purpose of capturing object movement with the self-recognizing technique. A camera can catch movement recording up to 2-3 min to prevent missing out on any major scene/incident.

Based on these KPPs for the camera, we have chosen three different types of cameras, NIR (near-IR, 750-1100 nm band), MWIR (mid-wave IR, 3-5mm) and LWIR (long-wave IR, 8-12 microns while choosing the cameras, we selected two fields of views, which are Wide field of view and pan-tilt.

\section{ELECTRONIC INTERFACE:}

Range: The range can be defined in the terms of the distance in which the camera will remain connected to the system. The range is given maximum priority because for longrange operations the connectivity to the system and LAN is important.

Speed: Speed or response time is particularly important as reaction time is directly proportional to early updates. The speed on most wireless networks (typically 1-54 Mbps) is far slower than even the slowest common wired networks (100Mbps up to several Gbps). However, in specialized environments, a wired network might be necessary.

Storage transfer rate: The transfer rate is the time required for transferring data from the camera system to storage, it depends on fast connectivity and strong network.

Multiple device connectivity: Wireless networks can connect to the multiple numbers of devices at the same time but in a wired network, additional devices require more wiring. Based on these $\operatorname{KPP}(s)$, the electronic interface has two potential interfaces: WIFI or USB cables. Pan-tilt units require a fixed mounting, whereas miniature nanny-cams with WIFI 
can be attached, removed, and replaced with Velcro or Picatinny Rail mounts so that different cameras can be used, and future cameras models can be incorporated.

\section{EXTENSION POLE:}

Weight: It should be lightweight and easy to carry during hostage/rescue operations.

Dimension: The diameter of the extension pole should be narrow to reach complex or protected areas. Extension pole's extended length should be able to reach longer lengths.

Flexibility: Camera extension pole should be bendable or flexible that allows it to follow through hidden areas (underneath of military vehicles, small holes, hazardous conditions, etc.) which are impossible to see through human eyes. The flexibility of the extension pole can be useful to get access to a wide variety of confined places.

Quick lever release: On any operation, it is important to assemble/configure the device as quickly as possible. Camera holder with the adjustable mounting base enables it to equip with either telescopic or snap together extension pole. Telescoping can be extended much faster with less difficulty than snap-together segments.

Angle of rotation: We can replace pan-tilt camera functions with a jolly stick controlled 360-degree probe to extend the existing capability of image coverage. Tungsten probe with coil prevents it from bending or permanent distortion which may be costly to maintain but it can be used on the long run.

Based on these KPPS, we have two Extension rod options: Telescoping which can be extended faster but is incompatible with cable from the camera to receiver, and Snaptogether that uses elastic cable between segments to facilitate alignment. Both approaches provide a 60 -inch extension without modification and collapse to under 14-inch length.

\section{Model Based Systems Engineering (MBSE Model):}

To create the DSM model, we have considered the findings based on the design requirements, KPP, MBSE pillars, and our research.

Our camera product development involves a type of camera (NIR, MWIR), the field of view (WFOV or Pan Tilt), extension type (telescoping or snap together), and interface type (WIFI or USB Cable).

Model-based system design (MBSE) has four different pillars: Performance, Components, Functional Behavior, and Engineering Analysis. A DSM model can be formed based on relations between these four pillars for our camera system as we have 3 different elements (Camera, Extension Pole, Electronic Interface) across functions, components, and performance. Table 1 shows this taxonomy. 
Rapp, Stephen; Witus, Gary; Kalgave, Shreya

\begin{tabular}{|c|c|c|c|}
\hline Subsystem & Functions: & Components: & Performance: \\
\hline $\begin{array}{l}\text { Electronic } \\
\text { Interface } \\
\text { WIFI }\end{array}$ & $\begin{array}{c}\text { Fast Local server } \\
\text { transfer (WLAN) } \\
\text { Data Cloud }\end{array}$ & $\begin{array}{l}\text { Inbuilt Interface } \\
\text { with Camera } \\
\text { System }\end{array}$ & \\
\hline USB & $\begin{array}{l}\text { Compatible FOV } \\
\text { up to 90-degree } \\
\text { Motion Detection } \\
\text { Removable SD } \\
\text { Easy Charging }\end{array}$ & $\begin{array}{l}\text { Memory Card } \\
\text { Storage, } \\
\text { Charging device, } \\
\text { OS Support } \\
\text { package }\end{array}$ & \\
\hline $\begin{array}{l}\text { Extension } \\
\text { Rod } \\
\text { Telescopic }\end{array}$ & $\begin{array}{l}\text { Contract/Extend } \\
\text { Versatility (108- } \\
\text { degree movement) } \\
\text { Quick Release } \\
\text { Lever Flexibility }\end{array}$ & $\begin{array}{l}\text { Pole/Cable } \\
\text { Hinge Rod } \\
\text { Clasp } \\
\text { Rotating Tip } \\
\text { Grip Handles }\end{array}$ & $\begin{array}{c}\text { Weight, Angle of } \\
\text { Rotation, Wireless } \\
\text { Control, Holder Shape, } \\
\text { Pole length }\end{array}$ \\
\hline $\begin{array}{c}\text { Snap } \\
\text { Together }\end{array}$ & $\begin{array}{c}\text { Contraction } \\
\text { /Extension } \\
\text { Twisting } \\
\text { Multi-angle view }\end{array}$ & $\begin{array}{c}\text { Pole } \\
\text { Hinge rod } \\
\text { Threaded End } \\
\text { Round Slider } \\
\text { Fluted grip } \\
\end{array}$ & $\begin{array}{c}\text { Weight } \\
\text { Angle of Rotation } \\
\text { Wireless Control } \\
\text { Holder Shape in inches } \\
\text { Length of Pole }\end{array}$ \\
\hline $\begin{array}{l}\text { Camera } \\
\text { Type } \\
\text { (All Options } \\
\text { - NIR and } \\
\text { MWIR) }\end{array}$ & $\begin{array}{c}\text { Video/Audio } \\
\text { Vision and Motion } \\
\text { Detection } \\
\text { Night Visio/Facial } \\
\text { Recognition Low } \\
\text { Light Sensitivity } \\
\text { Hi-res recording } \\
\end{array}$ & $\begin{array}{c}\text { Lens } \\
\text { Viewfinder } \\
\text { Image Sensor } \\
\text { Aperture } \\
\text { Flash }\end{array}$ & $\begin{array}{c}\text { Pixel Size, Video } \\
\text { Resolution and Format, } \\
\text { Image Ratio, } \\
\text { OS Support, Battery } \\
\text { Capacity, Memory, } \\
\text { Standby/Charging, } \\
\text { Dimensions } \\
\end{array}$ \\
\hline
\end{tabular}

Table 1: MSBE pillars classification for the PEC

Our DSM matrix is based on these element functions for the camera with the audio/video recording function directly connected to the USB which is the removable micro SD storage or fast local area server WLAN also with Cloud Data Storage. Vision and motion detection push the notification function of the camera whenever there is a change in state of the electronic interface system or the multi-angle view of the snap-together extension system The subsystem is optimally designed for its function, and that failure of a subsystem affects only one function. These are just a few examples that we considered while designing a DSM matrix based on the functional behavior of each element.

For the components, each camera, extension rod, and electronic interface are separate subsystem options each with their own dependencies to other subsystem options.

For example, In the camera system's components, Lens and Image sensors are directly connected to the electronic interface system's component. The Flash of the camera system is also connected to the charging device and the battery of its own. Which gives the link between the camera system's component pillar and performance pillar. Aperture is also dependent on the extension pole as it would affect the focal length of the camera.

These three pillars have many interlinks between each other. MBSE helped with Component-Based DSM by detecting defects earlier in the process and allowed the best 
camera product to build. The DSM is made using the links between the pillars and on assumptions made in the research mentioned above to get to Epoch B.

Table 2 below shows all the technology options to be traded and used in the DSM.

\begin{tabular}{|c|l|l|}
\hline 1 & WIFI & Goes with all except pan Tilt field of view \\
\hline 2 & USB & $\begin{array}{l}\text { Goes with all except NIR-WFOV because NIR cameras are } \\
\text { easier to go with WIFI. Goes with NIR pan tilt(Pan tilt } \\
\text { compulsory needs USB) }\end{array}$ \\
\hline 3 & Telescoping & $\begin{array}{l}\text { Goes with all and they can have USB cable running through } \\
\text { them }\end{array}$ \\
\hline 4 & $\begin{array}{l}\text { Snap } \\
\text { together }\end{array}$ & $\begin{array}{l}\text { Goes with all except pant tilt camera as it needs Telescoping } \\
\text { only(Snap together can only work for small cameras works } \\
\text { only with WFOV) }\end{array}$ \\
\hline 5 & Pan Tilt & $\begin{array}{l}\text { Pan tilt cameras are heavy, they only go with telescoping } \\
\text { and USB }\end{array}$ \\
\hline 6 & WFOV & $\begin{array}{l}\text { WFOV both USB and WIFI } \\
\text { NIR Goes with WIFI and USB but easier with WIFI ( but } \\
\text { also can go with USB for only this option NIR-Pan Tilt) }\end{array}$ \\
\hline 7 & NIR &
\end{tabular}

Table 2: Technology assumptions for the PEC Example

Our DSM model is a product type components-based DSM as we have three different elements integrated as a core system. It can also be considered as an Information Component-based DSM as there is an exchange of information between elements from camera to extension poles to USB or Wi-Fi or vice versa.

Our model has four epochs and we eliminate the options at each epoch to get the final set of designs for PD. Our camera can be NIR or MWIR with either WFOV or Pan Tilt, extension type is either telescoping or snap together, and interface type is WIFI or USB. Based on these options, we have 16 possible system combinations at epoch A. We reduce the designs with assumptions (Table 2) based on improved information going forward.

Figure 3 below shows the SBD DSM with the technology options and their dependencies for the PEC system. The sequence lines represent options from 1 to 8 . For example, let's consider options for snap together, and telescoping (IDs 3 and 4) is compatible with both the WIFI and USB cable (ID 1 and 2). Also, if we look at WIFI, it is compatible with Telescoping, Snap Together, NIR-WFOV, MWIR-WFOV (IDs 3, 4, 5, and 7).

\begin{tabular}{|l|l|l|l|l|l|l|l|r|r|}
\hline NAME & ID & 1 & 2 & 3 & 4 & 5 & 6 & 7 & 8 \\
\hline WIFI & 1 & & & 1 & 1 & 1 & & 1 & \\
\hline USB Cable & 2 & & & 1 & 1 & 1 & 1 & 1 & 1 \\
\hline Telescoping & 3 & 1 & 1 & & & 1 & 1 & 1 & 1 \\
\hline Snap Together & 4 & 1 & 1 & & & 1 & & 1 & \\
\hline NIR-WFOV & 5 & 1 & 1 & 1 & 1 & & & & \\
\hline NIR-Pantilt & 6 & & 1 & 1 & & & & & \\
\hline MWIR-WFOV & 7 & 1 & 1 & 1 & 1 & & & & \\
\hline MWIR-Pantilt & 8 & & 1 & 1 & & & & & \\
\hline
\end{tabular}

Figure 3: SBD DSM and their Dependencies for the PEC system 
This DSM gives us an idea about the sets of possible designs by showing the dependencies but at the option level. We have found ten combinations at Epoch B that are compatible with our camera product, that would be built and utilized by customers (beta phase of the product cycle).

Set-Based Design helps us to eliminate the less efficient set of solutions and to choose the most efficient set solutions at any given epoch. It should be noted that this is typically done qualitatively with both large automotive and defense enterprises, including Toyota, but it is only recently that consideration has been given to exploit system integration burden in a quantitative manner using both SBD and DSM.

\begin{tabular}{|c|c|c|c|}
\hline EPOCH A & EPOCH B & EPOCH $\Gamma$ & EPOCH $\Omega$ \\
\hline NIR-WFOV-Telescoping-WIFI & Stays in & Stays in & Stays in \\
\hline NIR-WFOV-Telescoping-USB & Stays in & Stays in & Stays in \\
\hline NIR-WFOV-Snap Together-WIFI & Stays in & Stays in & Drops Out \\
\hline NIR-WFOV-Snap Together-USB & Stays in & Stays in & Drops Out \\
\hline NIR-Pan Tilt-Telescoping-WIFI & Stays in & Drops Out & Drops Out \\
\hline NIR-Pan Tilt-Telescoping-USB & Stays in & Drops Out & Drops Out \\
\hline NIR-Pan Tilt-Snap Together-WIFI & Stays in & Drops Out & Drops Out \\
\hline NIR-Pan Tilt-Snap Together-USB & Stays in & Drops Out & Drops Out \\
\hline MWIR-WFOV-Telescoping-WIFI & Stays in & Drops Out & Drops Out \\
\hline MWIR-WFOV-Telescoping-USB & Stays in & Drops Out & Drops Out \\
\hline MWIR-WFOV-Snap Together-WIFI & Drops Out & Drops Out & Drops Out \\
\hline MWIR-WFOV-Snap Together-USB & Drops Out & Drops Out & Drops Out \\
\hline MWIR-Pan Tilt-Telescoping-WIFI & Drops Out & Drops Out & Drops Out \\
\hline
\end{tabular}

Table 3: Epochs for camera system

Epoch $\Gamma$ is found using the DSM clustering to find the best fit after each combination was analyzed. Figure 4 below shows the DSM clustering results and notation.

\begin{tabular}{|c|c|c|c|c|c|c|c|c|c|c|}
\hline NAME & ID & 1 & 2 & 3 & 4 & 5 & 6 & 7 & 8 & $\sum_{k=1}^{n} A_{i j k}$ \\
\hline WIFI & 1 & & $4 / 6$ & $2 / 8$ & $2 / 6$ & $2 / 6$ & $1 / 5$ & $2 / 6$ & $1 / 5$ & $S C_{i j}=\overline{\sum_{k=1}^{n} B_{i j k}}$ \\
\hline USB Cable & 2 & $4 / 6$ & & $4 / 8$ & $2 / 8$ & $2 / 8$ & $1 / 7$ & $2 / 8$ & $1 / 7$ & \\
\hline Telescoping & 3 & $2 / 8$ & $4 / 8$ & & $4 / 6$ & $2 / 8$ & $1 / 7$ & $2 / 8$ & $1 / 7$ & $\mathrm{SC}_{\mathrm{ij}}$ : Simılarty Coefficient \\
\hline Snap Together & 4 & $2 / 6$ & $2 / 8$ & $4 / 6$ & & $2 / 6$ & $1 / 5$ & $2 / 6$ & $1 / 5$ & 1: row \\
\hline NIR-WFOV & 5 & $2 / 6$ & $2 / 8$ & $2 / 8$ & $2 / 6$ & & $2 / 4$ & $4 / 4$ & $2 / 4$ & j: Column \\
\hline NIR-Pantilt & 6 & $1 / 5$ & $1 / 7$ & $1 / 7$ & $1 / 5$ & $2 / 4$ & & $2 / 4$ & $2 / 2$ & k: Number of common elements \\
\hline MWIR-WFOV & 7 & $2 / 6$ & $2 / 8$ & $2 / 8$ & $2 / 6$ & $4 / 4$ & $2 / 4$ & & $2 / 4$ & $\mathrm{~A}_{\mathrm{ijk}}$ : Common elements \\
\hline MWIR-Pantilt & 8 & $1 / 5$ & $1 / 7$ & $1 / 7$ & $1 / 5$ & $2 / 4$ & $2 / 2$ & $2 / 4$ & & $B_{i j k}:$ Total elements \\
\hline
\end{tabular}

Figure 4: SBD DSM clustering for the PEC system

Moving ahead from Epoch B (10 combination design options) to Epoch $\Gamma$ (4 combination design options), we have used the DSM based on similarity coefficient method clustering algorithm (Figure 4) to narrow down the options. Epoch $\Gamma$ options are NIR-WFOVTelescoping-USB, NIR-WFOV-Snap Together-WIFI, MWIR-WFOV-Telescoping-USB, MWIR-WFOV-Snap together-WIFI.

We have chosen these options by comparing one element with its possible compatible elements. We consider the electronic interface WIFI's two options for the extension rod: telescoping and snap together. Then we compare the combination of WIFI with telescoping and snap together, Look at $\mathrm{SC}_{13}(2 / 8)$ and $\mathrm{SC}_{14}(2 / 6)$ in Figure 4. 
We have chosen WIFI and snap together combination over WIFI and telescoping combination because $\mathrm{SC}_{14}>\mathrm{SC}_{13}$. $\mathrm{SC}_{14}$ denotes for WIFI and Snap together there are total 6 elements and out of these 6 elements, they are common in two elements. If the SC is the same for two combinations, then we will reconsider the assumptions.

At Epoch $\Omega$ phase (Table 3) we have chosen three combinations based upon the cost effectiveness and low light sensitivity.

\section{Conclusion:}

This study shows a framework to integrate Dependency and Structure Modeling into SBD using the DSM tool. The example considered design requirements to form a DSM model covering subsystem options to create a set solution for PD. While temporally passing through the PD epochs, we showed notional updated decisions based on reviewing the DSM at the epochs. Epoch A started with 13 options and were reduced to 10 in Epoch B. The dependency analysis supported this reduction. At Epoch $\Gamma$ we reduced the options to 4 using DSM clustering. Based on cost and requirements we down selected to two final designs. This framework integrates DSM into SBD to reduce uncertainty and risk.

\section{References:}

Browning, T. (2015). Modeling and managing complex systems, Proceedings of the 17th International DSM Conference, Fort Worth (Texas, USA), 4-6 November 2015. München: Hanser. doi: 0.1109/TEM.2015.2491283

Doerry, N. (2009). Using the Design Structure Matrix to Plan Complex Design Projects, Conference: ASNE Intelligent Ships Symposium, Philadelphia (Pennsylvania, USA), 20 May 2009.

McKenney, T., Kemink, L., \& Singer, D. (2011). Adapting to changes in design requirements using set-based design. Naval Engineers Journal, 123(3), 67-77.

Rapp, S., Doerry, N., Chinnam, R., Monplaisir, L., Murat, A., Witus, G. (2018). Set-Based Design and Optimization.... can they live together and make better Trade Space Decisions?, NDIA Ground Vehicle Systems Engineering and Technology Symposium, Novi (Michigan, USA), 7 - 9 August 2018.

Rapp, S. (2017). Product Development Resilience through Set-Based Design, Dissertation, Digital Commons @ Wayne State University, 2017. doi: 10.13140/RG.2.2.22691.14885

Singer, D., Doerry, N., \& Buckley, M. E. (2009). What Is Set-Based Design? Naval Engineers Journal, 121(4), 31-43.

Sobek, D. II, Ward, A., Liker, J. (1999). Toyota's Principles of Set-Based Concurrent Engineering. Sloan Management Review, Winter 1999; 40, 2.

Contact: Stephen Rapp, U.S. Army CCDC - Ground Vehicle Systems Center, Analytics, 650111 Mile Rd, Warren, MI USA 48397-5000, +1 586.282.1450, stephen.h.rapp.civ@ mail.mil 